\section{Digital gangrene in small cell lung cancer: response to aspirin treatment}

\author{
J E Arrowsmith, M A Woodhead, \\ D H Bevan, E M Nanson, A R C Cummin
}

\begin{abstract}
A patient who had small cell lung cancer complicated by symmetrical peripheral gangrene, secondary to spontaneous platelet aggregation, improved dramatically after starting aspirin treatment.
\end{abstract}

There are many documented non-metastatic effects of malignancy. The association between malignant disease and disorders of haemostasis is well established. ${ }^{1-7}$ Digital gangrene may be associated with malignancies occurring in the nasopharynx, stomach, colon, breast, ovary, uterus, and kidney, ${ }^{8}$ but has rarely been described in bronchial malignancy. ${ }^{89}$ We report a patient with disseminated bronchial malignancy who presented with symmetrical digital gangrene, due to spontaneous platelet aggregation, which responded to aspirin treatment.

\section{Case report}

A 63 year old man presented with a four week history of persistent fingertip pain and a two week history of symmetrical discoloration and ulceration of the tips of all the digits of both hands. He also had painless symmetrical discoloration of all toes. There was no history of Raynaud's phenomenon and no relation between the onset of symptoms and exposure to cold. Six months before this presentation he had been diagnosed as having carcinoma of the bronchus by bronchial biopsy. Although on review the histological features were those of small cell carcinoma, the biopsy specimens were originally reported as showing moderately differentiated squamous cell carcinoma. The patient gave a history of hypertension but was taking no regular medication. $\mathrm{He}$ had smoked 30 cigarettes daily before diagnosis.

On examination the patient appeared well. There were bilateral palmar Dupuytren's contractures but no clubbing or lymphadenopathy. Symmetrical cyanosis and gangrene of the tips of the fingers and thumb of both hands were present (figure). Clinical features of scleroderma were absent. All upper and lower limb pulses were palpable, including most of the digital pulses of the upper limbs. The tips of all the toes were cyanosed but did not show ischaemic changes. A hard, irregular liver was palpable in the right hypochondrium. There was no evidence of peripheral or central venous thrombosis.

Laboratory investigations gave the following results: haemoglobin $16.8 \mathrm{~g} / \mathrm{dl}$, packed cell volume 0.50 , erythrocyte sedimentation rate $3 \mathrm{~mm}$ in one hour. The white cell $(10.8 \times$ $\left.10^{9} / 1\right)$ and platelet counts $\left(336 \times 10^{9} / 1\right)$ were normal, as were the prothrombin time, activated partial thromboplastin time, and thrombin clotting times and serum electrolytes, urea, creatinine, glucose, and thyroxine concentrations. Liver function tests gave abnormal results: alkaline phosphatase 233 (normal range (NR) 30-120) IU/l, alanine transaminase $(\mathrm{NR}<45) 50 \mathrm{IU} / \mathrm{l}$, and $\gamma$ glutamyl transferase 184 (NR $<60$ ) IU/1. C reactive protein was $9(\mathrm{NR}<8.0) \mathrm{mg} / \mathrm{l}$. Total plasma protein concentrations were within normal limits; electrophoresis showed raised $\alpha_{2}$ and gamma globulins. Neither paraprotein nor cryoglobulin was detected. Abdominal ultrasound indicated multiple echogenic lesions within the liver consistent with metastases. Radiographs of both hands showed loss of soft tissue at the tips of the digits but no bone resorption. The chest radiograph showed right paratracheal and hilar masses with left apical shadowing and destruction of the posterior end of the fourth left rib. Echocardiography showed aortic valve sclerosis but no evidence of endocarditis.

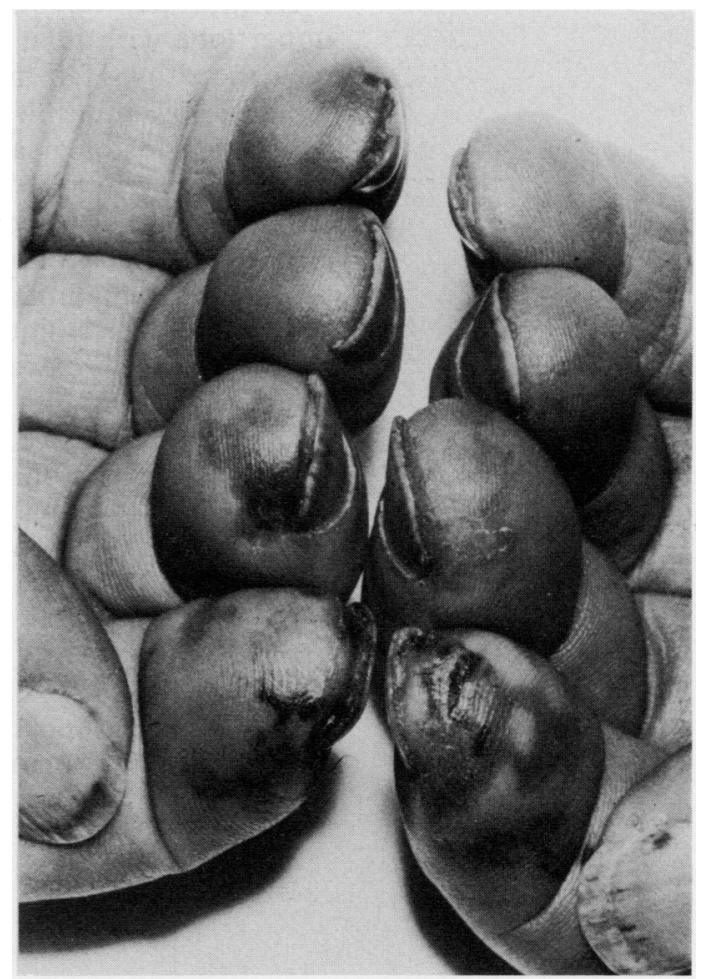

The patient's digital vasculitis, with lesions under the thumb nails. 
Plasma viscosity was slightly increased at 1.42 (NR < 1.35) mPa.s. Whole blood viscosity was also slightly increased at both high $(94.5 / \mathrm{s})$ and low $(0.94 / \mathrm{s})$ shear rates $(6.47 \mathrm{mPa} . \mathrm{s}$ and $45.4 \mathrm{mPa}$.s respectively). Testing of Hep2 cell substrate for centromere specific antinuclear antibody gave a strongly positive result with a titre greater than $1 / 2560$. Other autoantibodies, including anticardiolipin and antidouble stranded DNA, were not detected. Concentrations of both IgG and IgM circulating immune complexes were slightly raised at $94(\mathrm{NR}<84)$ and $155(\mathrm{NR}<110) \mathrm{ng} / \mathrm{l}$ respectively.

Platelet rich plasma from the patient, when stirred in an aggregometer cuvette at $37^{\circ} \mathrm{C}$, showed spontaneous platelet aggregation, which reached completion at 6 minutes. Aggregation in response to adenosine diphosphate, ristocetin, collagen, and arachidonate was normal. Circulating in vivo platelet aggregates were not detected in blood from the antecubital vein by the method of $\mathrm{Wu}$ and Hoak. $^{10}$

Platelet poor plasma from the patient did not cause aggregation of plasma rich protein or washed platelets from normal donors. The patient's plasma had normal concentrations of antithrombin III, protein C, and free protein $S$. The concentration and multimeric composition of von Willebrand factor in both plasma and platelets were normal.

After a week's treatment with aspirin $300 \mathrm{mg}$ daily spontaneous platelet aggregation was abolished and the patient's symptoms rapidly and dramatically resolved. In view of a maintained response to aspirin he was discharged home four weeks after admission.

A week later, however, he was readmitted with a 24 hour history of severe abdominal pain and died 48 hours later.

At necropsy tumour was seen in the right upper lobe with hilar and porta hepatis lymphadenopathy. Half the liver parenchyma was replaced by multiple umbilicated tumours, which were histologically found to be small cell anaplastic carcinoma. Two large punched out, chronic posterior ulcers were found in the first part of the duodenum, which may have explained the abdominal pain. There was no evidence of perforation, haemorrhage, or peritonitis. There was severe atheromatous disease of the coronary arteries and aorta but no macroscopic evidence of thrombus formation or valvular vegetation. Histological sections of the affected fingertips showed no evidence of vasculitis, vascular obstruction, or malignant cells.

\section{Discussion}

Since Trousseau spoke about it, ${ }^{3}$ disruption of the haemostatic system resulting in thrombosis has been recognised as a common paraneoplastic syndrome. Circulating tumour products activate various haemostatic mechanisms, including platelets. ${ }^{56} \mathrm{Clinical}$ lesions, however, directly attributable to enhanced platelet aggregation, as here, have not been reported before.

In this patient florid signs of obstructive small vessel disease were associated with spontaneous platelet aggregation, a phenomenon which indicates platelet hyperreactivity. Aspirin abolished spontaneous platelet aggregation and improved symptoms, suggesting that platelet hyperreactivity was important in the pathogenesis of the digital gangrene. Local factors in the digital vessels may also have played a part, because in vivo platelet clumps were not found in blood taken from the antecubital vein. Similar lesions due to platelet aggregation are a classical presenting sign in essential thrombocythaemia, a myeloproliferative disease with hyperreactive platelets.

We failed to detect circulating platelet aggregating activity in cell free plasma. Abnormal von Willebrand factor, sometimes a cause of spontaneous platelet aggregation, was not found. Immune complexes may cause platelet aggregation, but if those present in this case were responsible the patient's plasma might have been expected to aggregate control platelets. These findings suggest that the abnormal stimulus to aggregation in this case was not free in plasma but closely associated with platelet membranes. Membrane phospholipids from tumour cells would fit this role.

In a previously reported case digital ischaemia associated with small cell carcinoma of the lung was attributed to vasospasm. ${ }^{7}$ In our patient a platelet mediated mechanism is likely to have been the cause of the digitial gangrene.

1 Milroy R, Douglas JT, Campbell J, Carter R, Lowe GDO, Banham SW. Abnormal haemostasis in small cell lung cancer. Thorax 1988;43:978-81.

2 Hagedorn AB, Bowie EJW, Elveback LR, Owen CA Jr. Coagulation abnormalities in patients with inoperable lung cancer. Mayo Clin Proc 1974;49:647-53.

3 Trousseau A. Clinique médicale de l'Hôtel Dieu de Paris. 2nd ed. Paris, 1865.

4 Sun NCJ, McAfee WM, Hum GJ, Weiner JM. Haemostatic abnormalities in malignancy, a prospective study of one hundred and eight patients. Am J Clin Pathol 1979;71: 10-6.

5 Jamieson GA, Bastida E, Ordinas A. Mechanisms of platelet aggregation by human tumor cell lines. Prog Clin Biol Res 1982;89:405-13.

$6 \mathrm{Al}-\mathrm{Mondhiry} \mathrm{H}$. Beta-thromboglobulin and platelet factor 4 in patients with cancer: correlation with the stage of disease and the effect of chemotherapy. Am J Haematol 1983;14:105-11.

7 Field J, Lane IF. Carcinoma of the lung presenting with digital ischaemia. Thorax 1986;41:573-4.

8 Hawley PR, Johnston AW, Rankin JT. Association between Digital Ischaemia and Malignant Disease. $\mathrm{Br}$ Med J 1967; iii:208-12.

9 Domz CA, Chapman CG. Pseudo Raynaud's: cryoglobulinemia secondary to occult neoplasm. Calif Med 1961; 95:391-3.

$10 \mathrm{Wu} \mathrm{KK}$, Hoak JC. A new method for the quantitative detection of platelet aggregates in patients with arterial insufficiency. Lancet 1974; ii:924-6. 